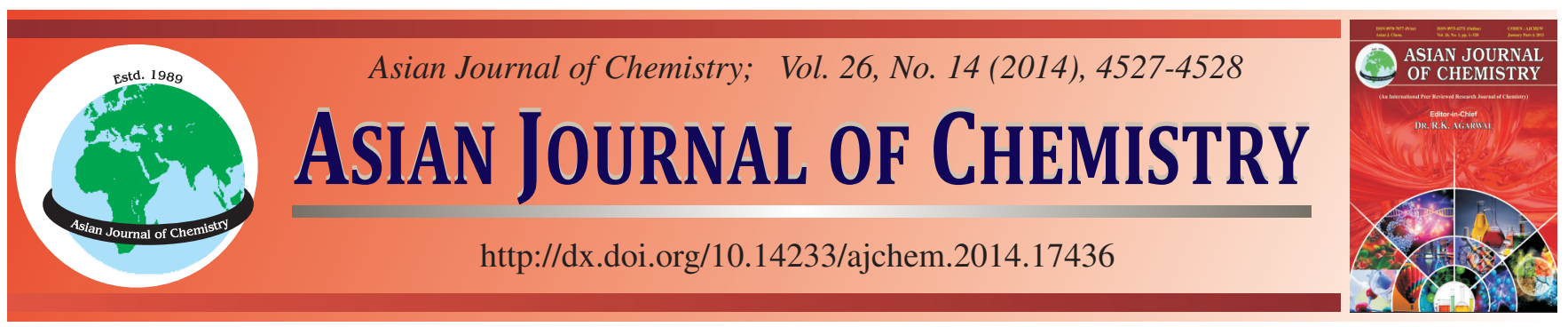

NOTE

\title{
A New Zinc(II) Coordination Polymer with Two-Dimensional sql Topology Based on Asymmetrically Tricarboxylate and Nitrogen-Containing Ligand
}

\section{LIPING XU}

Department of Chemistry Engineering, Zibo Vocational Institute, Shandong 255314, P.R. China

Corresponding author: E-mail: dzjmsheng@163.com

Received: 21 February 2014;

Accepted: 30 April 2014;

Published online: 5 July 2014;

AJC-15515

\begin{abstract}
A new coordination polymer $\left\{\left[\mathrm{Zn}(\mathrm{Hbtc})(\mathrm{bpmp})_{0.5}\right] \cdot \mathrm{H}_{2} \mathrm{O}_{\mathrm{n}}\right\}(\mathbf{1})\left[\mathrm{H}_{3}\right.$ btc=biphenyl-3,4',5-tricarboxylate and bpmp=1,4-bis (4-pyridylmethyl)piperazine] has been obtained under hydrothermal conditions. Complex $\left\{\left[\mathrm{Zn}(\mathrm{Hbtc})(\mathrm{bpmp})_{0.5}\right] \cdot \mathrm{H}_{2} \mathrm{O}_{\mathrm{n}}\right\}(\mathbf{1})$, is triclinic, space group P-1 with $\mathrm{a}=8.189$ (5) $\AA, \mathrm{b}=8.919$ (5) $\AA, \mathrm{c}=15.998(5) \AA, \beta=90.622(5)^{\circ} \mathrm{V}=1050.9(9) \AA^{3}, \mathrm{Z}=2, \mathrm{M}_{\mathrm{r}}=501.78, \mathrm{D}_{\mathrm{c}}=1.586 \mathrm{~g} / \mathrm{cm}^{3}, \mathrm{~F}(000)$ $=516$ and $\mu=1.22 \mathrm{~mm}^{-1}$. The final refinement gave $\mathrm{R}=0.0483$ and $w \mathrm{R}=0.1187$ for 2390 reflections with $\mathrm{I}>2 \sigma$ (I). X-ray diffraction analysis reveals that complex 1 shows a two-dimensional $(4,4)$ sql layer.
\end{abstract}

Keywords: Zinc(II), Coordination polymer, Topology.

In recent years, the study of design and construction of coordination polymers via careful selection of metal ions and organic ligands has attracted much attention due to their structural diversity and the potential applications in adsorption, magnetism, separation ${ }^{1-4}$, catalysis, optics, etc. Generally, structures and properties of coordination polymers are affected by many factors, mainly including the organic ligands with suitable functional groups and molecular skeletons, nature of the metal ions and reaction conditions such as temperature, $\mathrm{pH}$ value, solvent, $e t c^{5,6}$.

In this paper, we used the asymmetrically tricarboxylate and pyridyl-containing ligands to construct a new $\mathrm{Zn}$ (II) coordination polymer.

All the reagents and solvents employed were commercially available and used without further purification. Elemental analysis was carried out on a Vario ELIII elemental analyzer. or. FT-IR spectra were recorded with a Bruker Equinox 55 FT-IR spectrometer as a dry $\mathrm{KBr}$ pellet in the $4000-400 \mathrm{~cm}^{-1}$ range.

Preparation of $\left\{\left[\mathbf{Z n}(\mathbf{H b t c})(\mathbf{b p m p})_{0.5}\right] \cdot \mathbf{H}_{2} \mathbf{O}_{\mathbf{n}}\right\}$ (1): The starting materials were biphenyl-3,4',5- tricarboxylate $\left(\mathrm{H}_{3} \mathrm{btc}\right)$ (0.143 g, $0.5 \mathrm{mmol}), 1,4-b i s(4-p y r i d y l m e t h y)$ piperazine (bmpm) (0.105 g, $0.5 \mathrm{mmol}),(0.145 \mathrm{~g}, 0.5 \mathrm{mmol}), \mathrm{NaOH}$ $(0.04 \mathrm{~g}, 1 \mathrm{mmol})$ and $\mathrm{H}_{2} \mathrm{O}(15 \mathrm{~mL})$. Colorless crystals of complex 1 were collected in $45 \%$ yield based on $\mathrm{Zn}$. Anal. Calcd. for: C, 50.27; H, 2.61; N, 5.58. Found: C, 50.24; H, 2.63; N, 5.61. IR (KBr, $\left.v_{\max }, \mathrm{cm}^{-1}\right): 1620$ (s), 1530 (s), 1503 (s), 1437 (s), 1347 (m), 1263 (m), 1217 (m), 1179 (w), 1047 (m), 955 (w), 836 (m), 797 (m).

X-ray crystallography: Single crystal X-ray diffraction analyses of complex $\mathbf{1}$ was carried out on a Bruker SMART APEXII CCD diffractometer equipped with a graphite monochromated $\mathrm{MoK}_{\alpha}$ radiation $(\lambda=0.71073 \AA$ ) by using a $\omega$-scan mode. Empirical absorption correction was applied using the SADABS programs ${ }^{7}$. All the structures were solved by direct methods and refined by full-matrix least-squares methods on $\mathrm{F}^{2}$ using the program SHEXL $97^{8}$. All non-hydrogen atoms were refined anisotropically. The hydrogen atoms were located by geometrically calculations and their positions and thermal parameters were fixed during the structure refinement.

Single-crystal X-ray diffraction analysis reveals that the asymmetric unit of complex 1 consists of one crystallographically independent $\mathrm{Zn}$ (II) ion, one $\mathrm{Hbtc}^{2-}$, half of bmpm ligand and one lattice water molecule. The center $\mathrm{Zn}$ (II) atom is in a tetrahedral coordination environment having three Zn-O bond with carboxylate groups[1.939(3)-2.113(3) $\mathrm{\AA}]$ (Fig. 1) and one Zn-N bond from pyridyl ligand [ $\mathrm{Zn}(1)-\mathrm{N}(1)=$ 2.048 (4) А̊]. The Hbtc ${ }^{2-}$ ligands adopt $\mu_{1}-\eta^{1}: \eta^{0}$ and $\mu_{2}-\eta^{1}: \eta^{1}$ coordination mode. The $\mu_{2}-\eta^{1}: \eta^{1}$ carboxylate groups connected $\mathrm{Zn}$ (II) ions to form a dinuclear $\mathrm{Zn}$ (II) unit with the $\mathrm{Zn}-\mathrm{Zn}$ distance of $4.266 \AA$. The dinuclear $\mathrm{Zn}$ (II) units connect by $\mathrm{Hbtc}^{2-}$ ligand to form a one-dimensional chains (Fig. 2). The $1 \mathrm{D}$ chains are further connected by bmpm ligands to construct two-dimensional layer structure (Fig. 3). 


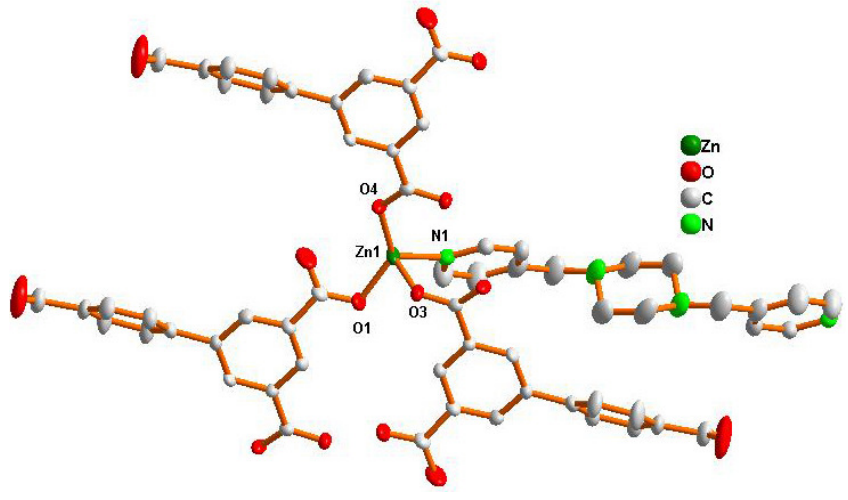

Fig. 1. A local coordination geometry of central $\mathrm{Zn}$ (II) atom

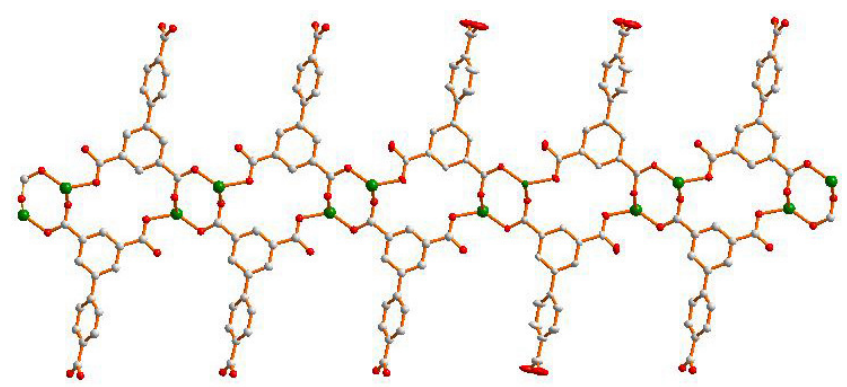

Fig. 2. 1D chain constructed by $\mathrm{Hbtc}^{2-}$ and $\mathrm{Zn}(\mathrm{II})$ ions

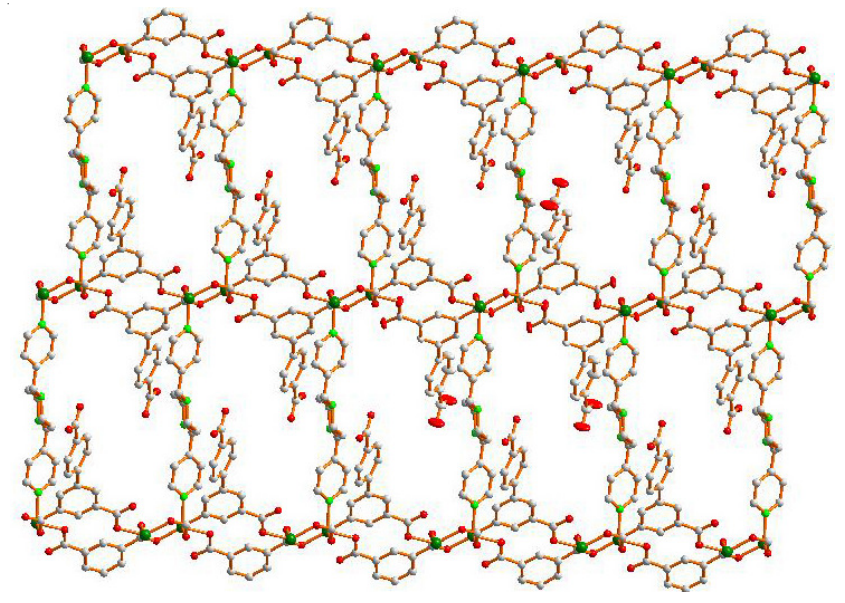

Fig. 3. 2D layer structure for complex 1

\section{REFERENCES}

1. M. O'Keeffe and O.M. Yaghi, Chem. Rev., 112, 675 (2012).

2. G.P. Yang, L. Hou, X.J. Luan, B. Wu and Y.Y. Wang, Chem. Soc. Rev., 41, 6992 (2012).

3. S.L. Cai, S.R. Zheng, J. Fan, J.B. Tan, T.T. Xiao and W.G.J. Zhang, Solid State Chem., 184, 3172 (2011).

4. J. Xu, Z. Su, M.S. Chen, S.S. Chen and W.Y. Sun, Inorg. Chim. Acta, 362, 4002 (2009).

5. S.L. Huang, L. Zhang, Y.J. Lin and G.X. Jin, Cryst. Eng. Comm., 15, 78 (2012).

6. Z.L. Chen, X.L. Liu, C.B. Zhang, Z. Zhang and F.P. Liang, Dalton Trans., 40, 1911 (2011).

11. SAINT Software Reference Manual; Bruker AXS: Madison, WI (1998).

12. G.M. Sheldrick, SHELXTL NT, version 5.1; Program for Solution and Refinement of Crystal Structures, University of Göttingen, Germany (1997). 